\title{
EMD-DF64: A 64-Bit File Format for Energy Monitoring and Disaggregation Datasets
}

\author{
Lucas Pereira $^{1,2,{ }^{*}}$, Nuno Velosa ${ }^{1,3}$, and Manuel Pereira ${ }^{1,3}$ \\ ${ }^{1}$ ITI, LARSyS, Funchal, 9020-105, Portugal \\ ${ }^{2}$ Técnico Lisboa, Lisbon, 1049-001, Portugal \\ ${ }^{3}$ Universidade da Madeira, Funchal, 9020-105, Portugal \\ *corresponding author(s): Lucas Pereira (lucas.pereira@tecnico.ulisboa.pt)
}

\begin{abstract}
There is a consensus in the Non-Intrusive Load Monitoring research community on the importance of public datasets in furthering load disaggregation research. Nevertheless, despite the considerable efforts to release public data, not many steps have been taken towards homogenizing how these are made available to the community and providing seamless access to the available and future datasets. In this paper, we present the Energy Monitoring and Disaggregation Data Format (EMD-DF64). EMD-DF64 is a data model, file format, and application programming interface developed to provide a unique interface to create, manage, and access electric energy datasets. The proposed format is an extension of the well-known Sony WAVE64 format that supports the storage of audio data and metadata annotations in the same file, thus reducing the number of artifacts that have to be maintained by the dataset users.
\end{abstract}

\section{Introduction}

Non-Intrusive Load Monitoring (NILM), or more generally load/energy disaggregation, is a promising approach for enabling a cost-effective way of providing detailed information about the energy consumption of individual appliances ${ }^{1}$. The availability of public datasets opens new prospects for this technology, helping researchers create more systematic evaluation processes that can be used across different approaches, similar to what happened in other application domains of machine learning like face and speech recognition ${ }^{2}$.

Nevertheless, despite the tremendous efforts in releasing public datasets as reviewed in ${ }^{3,4}$, not many steps have been taken towards homogenizing the way these are made available and accessible to the community. In fact, Several data formats have been employed to store NILM datasets, with Comma-separated values (CSV) emerging has one of the most used formats given the simplicity of the data representation, e.g., ${ }^{5-7}$. Yet, many other formats were adopted, namely $\mathrm{HDF}^{8}$, relational databases $^{9}$, as well as audio/video formats such as FLAC $^{10,11}$, WAVE ${ }^{12,13}$, and Matroska Media Containers ${ }^{14}$. A direct consequence of the variety of data formats is that in order to use a dataset, researchers first have to understand the underlying structure of the data and produce code to interface with their algorithms. In fact, in ${ }^{15}$ the authors have identified the lack of convergence regarding a storage file format as one of the main challenges to NILM dataset interoperability.

Against this background, some research efforts have been devoted to homogenize existing datasets and provide a single interface to run evaluations. Two examples of such efforts are the Metadata proposal for NILM datasets ${ }^{16}$, and the Non-Intrusive Load Monitoring Toolkit (NILMTK) ${ }^{17}$. The two projects have been combined to form the NILMTK-DF, the data format supported by NILMTK that relies on HDF5, and the metadata structure proposed in ${ }^{16}$. Another example is the NilmDB project $^{18}$, which provides a generalized user interface to access, query, and analyze large time-series datasets, including NILM data. The underlying timestamped data is organized hierarchically and must follow a data stream layout (the number of columns and corresponding data type) determined when a data stream is created. Besides the time series data, NilmDB also supports attaching metadata in the form of key/value pairs.

$\mathrm{In}^{19}$ we proposed the Energy Monitoring and Disaggregation Data Format (EMD-DF), a standard data model and file format developed with the intent to represent and store high-frequency datasets (i.e., sampling rate greater or equal to $1 \mathrm{~Hz}$ ). In EMD-DF, the datasets are represented using the well-known Waveform Audio File Format (WAVE ${ }^{1}$ ). The idea of using an audio-based format to represent electric energy data was inspired by previous works where sound-cards are used to perform the acquisition of the current and voltage signals ${ }^{10,20}$, and have been shown to perform considerably well given the similarities to electricity waveforms ${ }^{21}$. Furthermore, being an extension of the Resource Interchange File Format (RIFF ${ }^{2}$ ), the WAVE format

\footnotetext{
${ }^{1}$ WAVE file format: http://fileformats.archiveteam.org/wiki/WAV

${ }^{2}$ RIFF file format, http://fileformats.archiveteam.org/wiki/RIFF
} 
has several properties that are desirable in the context of NILM datasets. More particularly:

1. The consumption data, ground-truth, and metadata are all stored in the same file, thus limiting the number of artifacts to be managed;

2. The resulting files are optimized to have very little overhead. Furthermore, since the sampling rate is fixed, only the initial timestamp is necessary to obtain the time of the remaining samples;

3. It is an uncompressed lossless format, i.e., all the original values of the data are kept untouched. Furthermore, it is fully compatible with audio-compression libraries such as WavePack ${ }^{3}$;

4. It is possible to extend the format at any time with additional chunks without breaking the file consistency, i.e., a WAVE file with additional chunks will consistently be recognized as a WAVE file. Hence backward compatibility with previously developed applications is guaranteed;

5. Finally, there is a diversity of mature programming interfaces to work with audio content, thus facilitating dataset manipulation and portability across different programming environments.

However, extending the WAVE file format comes with two major limitations:

1. To avoid having to store individual timestamps for each sample, the data must not contain missing values. Therefore, missing data is handled separately, either by: i) resampling whenever possible, i.e., when the number of missed samples is short and sparse, ii) break the datasets in different files when missing big blocks of data, and iii) resampling and breaking into multiple files when the missed data is both sparse and with large gaps.

2. The WAVE specification uses a 32-bit unsigned integer to represent the file size header. As such, dataset files are limited to a maximum of $4 \mathrm{~GB}$. This is equivalent to roughly 248 days of two 16-bit channels sampled at $50 \mathrm{~Hz}$. Still, there are datasets with sample rates in the order of $\mathrm{kHz}$. For example, BLUED ${ }^{22}$ was sampled at $12 \mathrm{kHz}$, meaning that each data file can only represent around 16 hours of the three 16-bit channels (i.e., two currents phases and one voltage phase).

In this paper, we present a 64-Bit version of EMD-DF (EMD-DF64) by including support to the Sony WAVE64 ${ }^{4}$ file format. Ultimately, this leads to a maximum file size of approximately 16 exabytes, which is equivalent to roughly 21 years of three 16-bits channels sampled at $4.3 \mathrm{GHz}$ ). Furthermore, EMD-DF64 was developed to support missing data by default, which is achieved by adding additional chunks to represent such gaps.

In the remainder of this paper, we first present a data model that supports EMD-DF64. Then, we present the data structure, which is an extension of the 64 bit Sony Wave64 file container. Then, we thoroughly describe how the data model and data structure are combined to form the EMD-DF64 file format. Finally, we discuss how the proposed data format contributes to the ongoing efforts to homogenized electricity consumption datasets.

\section{Methods}

\section{Data Model}

The data model that supports EMD-DF and EMD-DF64 is comprised of several data entities that should be present in a dataset to make it suitable for NILM research. Figure 1 shows an illustration of the proposed data model using the Unified Modeling Language $\left(\mathrm{UML}^{5}\right)$ notation. Overall, there are three main data entities: 1) consumption, 2) ground-truth, and 3) annotations. These are described next.

The consumption entity represents all the data elements that refer to energy consumption time-series. Consumption data can be of two different types: i) raw waveforms, i.e., current and voltage; or ii) processed waveforms, i.e., different metrics like real and reactive power. Furthermore, consumption data can represent both aggregated and disaggregated consumption. Finally, the latter can represent the consumption of individual appliances (e.g., a kettle) or an individual circuit (e.g., kitchen outlets that may or not contain the kettle). It is important to remark that all the entities that refer to consumption data are optional (cardinality of 0 or more) to cover as many variations of NILM datasets as possible. For example, BLUED contains only aggregated consumption data, while SustDataED ${ }^{13}$ contains aggregated and individual appliance consumption. Likewise, $\mathrm{RAE}^{23}$ contains data for aggregated, individual appliances and individual circuit consumption. Finally, it is also possible to find datasets that do not contain any form of aggregated consumption, e.g., PLAID ${ }^{24}$, Tracebase ${ }^{25}$ and GREEND ${ }^{26}$.

\footnotetext{
${ }^{3}$ WavPack Audio Compression, https://www . wavpack.com/

${ }^{4}$ Sony Wave64, http://fileformats.archiveteam.org/wiki/Sony_Wave64

${ }^{5}$ UML, https: / /www.uml.org/
} 


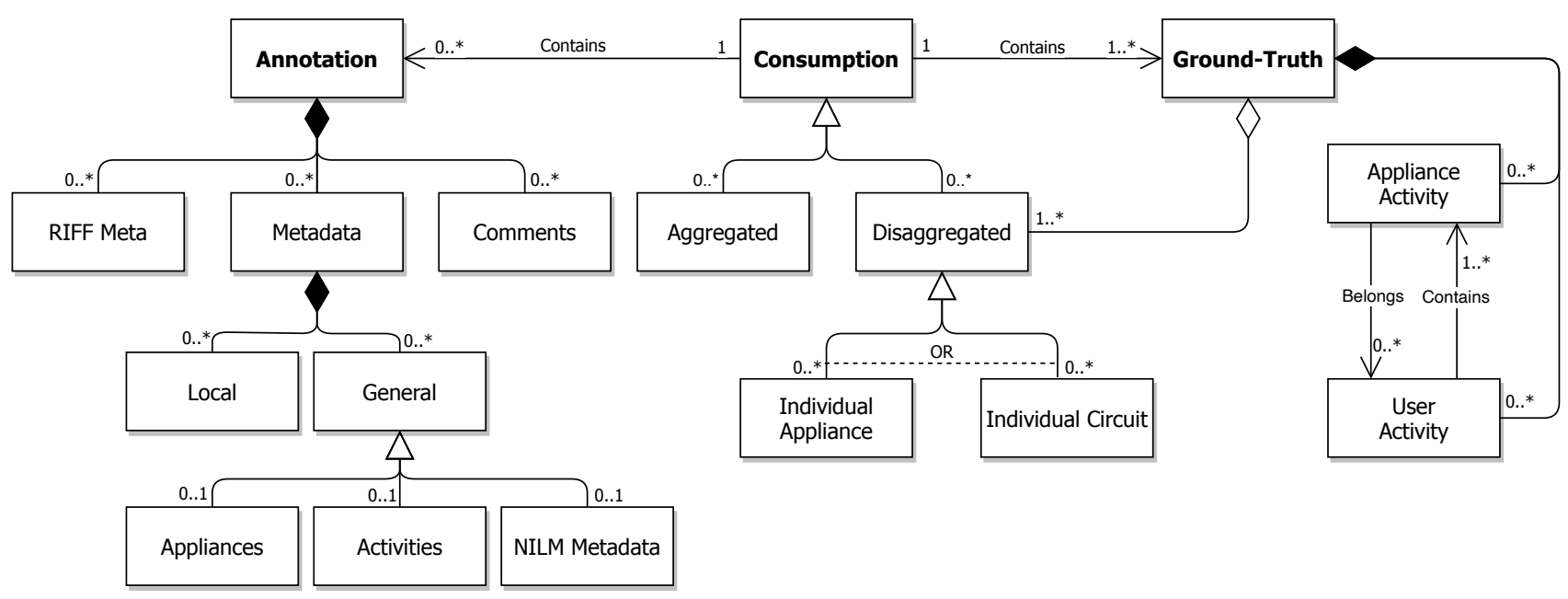

Figure 1. EMD-DF64: Data model overview

On the right-hand side of Figure 1 is the ground-truth entity. This entity is mandatory on a NILM dataset, and can be of four different types: i) individual appliance consumption, ii) individual circuit consumption, iii) appliance activity, and iv) user activity. Individual appliance and individual circuit consumption are a special type of consumption data used to train, test, and validate event-less approaches. On the other hand, appliance activities provide information about the power events that exist on a dataset and are required to train, validate, and test event-based approaches.

We have also introduced the concept of user activities, which is straightforward terms refer to actions that people perform involving the use of electric appliances, e.g., doing the laundry (involves clothes washer, clothes dryer, and iron) or preparing a meal (oven, stove, microwave, aid choppers, and blenders). Such user activities are important to evaluate different NILM application domains, e.g., Non-Intrusive Activity Detection (NIAD) ${ }^{27}$, and the detection of abnormal consumption behaviors ${ }^{28}$. It is important to note that one individual appliance activity can only be associated with one user activity (cardinality of 0 or 1 ). Otherwise, the total consumption of the user activities will be larger than the total consumption of the individual appliances, thus introducing inconsistency to the data model.

Lastly, we have the annotations entity. There are three types of optional annotations: 1) RIFF Meta, which are the default metadata chunks defined by RIFF, 2) comments, consisting of free text, and 3) user-defined metadata. The latter can be either which can be either local metadata, which refers to specific samples in the consumption data, or custom metadata annotations defined by the dataset creator and can serve multiple purposes. For example, in the current implementation, it is possible to provide a list with details for individual appliances and user activities and embed the annotations from the NILM metadata project.

\section{Data Structure}

In EMD-DF64, the different data entities are represented by extending the 64-bit Sony WAVE64 (SW64) file format. SW64 is an application of the RIFF in which the file contents are grouped and stored in separate chunks. Each chunk consists of three components: 1) chunk identifier - 128-bit globally unique identifier (GUID), e.g., "fmt " and "data"; 2) an unsigned, little-endian 64-bit integer representing the length of the chunk; 3) the chunk data. Finally, like all other RIFF-based formats, if a chunk's data size is not even, it is padded by 1 byte to make it so.

A WAVE64 file is composed of several chunks, four of which are mandatory. Furthermore, in a correctly formatted WAVE64 file, the first four bytes (GUID) must always spell out "riff" (lower-case). Some of the W64 chunks are briefly described in Table 1, with a particular focus on those reused in the EMD-DF data model. For more details about the RIFF standard, please refer to the original project documentation in $^{29}$.

The EMD-DF64 file format is based on 20 chunks. One directly inherited from the RIFF standard (Info), nine from the WAVE64 format (Format, Data, Cue, List, Associated Data List, Label, Note and Labeled Text Chunk), and the remaining 10 are custom chunks. The chunk structure of EMD-DF64 is illustrated in Figure 2. Table 2 provides a description of the 10 custom chunks.

\section{EMD-DF64 File Format Definition}

Next, we present how the different chunks that compose the EMD-DF64 data structure are combined to create a dataset. We first describe how the data format is defined in the Format and Config chunks. Then we show how the power measurements are 


\begin{tabular}{|c|c|c|c|c|}
\hline Name & GUID & Description & Parent & EMD-DF64 \\
\hline $\operatorname{RIFF}^{a}$ & 'riff' & This is the main chunk and is mandatory for every file that is based on the RIFF standard. & - & $\checkmark$ \\
\hline WAVE $^{a, b}$ & 'wave' & Identifies the contents of the RIFF chunk as being of the type w64. & riff & $\checkmark$ \\
\hline Format $^{a}$ & 'fmt' & Defines the data format, e.g. sampling rate, sample size and bits and the number of channels. & riff & $\checkmark$ \\
\hline Data $^{a}$ & 'data' & $\begin{array}{l}\text { Waveform data can be stored as a single contiguous array of interleaved samples or as a } \\
\text { discrete sequence of blocks of samples and silence wrapped in a 'wavl' chunk. }\end{array}$ & riff & $\checkmark$ \\
\hline Wave List & 'wavl' & Wraps sequences of data and silence chunks. & data & - \\
\hline Silent & 'slnt' & Represents silence and is defined as a count of silence samples. & wavl & - \\
\hline Fact & 'fact' & $\begin{array}{l}\text { Stores information about how the waveform data is organized. It is mandatory when the } \\
\text { waveform data is stored in a 'wavl' chunk and for all compressed audio formats. }\end{array}$ & riff & - \\
\hline Cue & 'cue' & $\begin{array}{l}\text { Identifies a series of positions in the waveform data as as having additional information } \\
\text { associated with them. There is at most one cue chunk per wave file, and it is followed by a list } \\
\text { of cue points. }\end{array}$ & riff & $\checkmark$ \\
\hline List & 'list' & This is a wrapper for RIFF chunks, which in the particular case of WAVE64 files is an associated data list ('adtl'). & riff & $\checkmark$ \\
\hline $\begin{array}{l}\text { Associated } \\
\text { Data List } \\
a, b\end{array}$ & 'adtl' & Identifies a list that contains individual information attached to the cue points defined in the cue chunk. & list & $\checkmark$ \\
\hline Label & 'labl' & Associates a text label to a specific cue point. Must be defined inside the associated data list chunk. & adtl & $\checkmark$ \\
\hline Note & 'note' & Same as label, but usually contains comment text for a specific cue point & adtl. & $\checkmark$ \\
\hline $\begin{array}{l}\text { Labeled } \\
\text { Text }\end{array}$ & 'ltxt' & $\begin{array}{l}\text { Associates a text comment to specific regions of waveform data. A region is a cue point whose } \\
\text { adtl list duration in samples is defined in this chunk. Must be defined inside the associated } \\
\text { data list chunk. }\end{array}$ & adtl & $\checkmark$ \\
\hline $\begin{array}{l}\text { Embedded } \\
\text { File Info }\end{array}$ & 'file' & $\begin{array}{l}\text { Contains information described in other file formats (e.g. ASCII text files) that is associated } \\
\text { with a particular cue point. }\end{array}$ & adtl & - \\
\hline Playlist & 'plst' & Specifies a play order for a series of cue points. & riff & - \\
\hline Info $^{b}$ & & Identifies a list that contains the info chunks ${ }^{29}$. & riff & $\checkmark$ \\
\hline
\end{tabular}

Table 1. List of chunks that compose the RIFF-WAVE64 file format

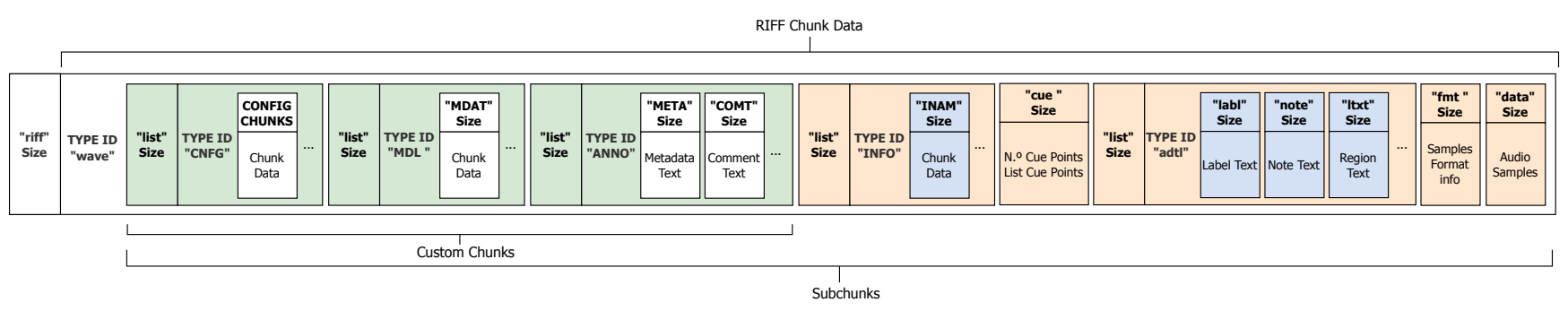

Figure 2. Chunk structure of the EMD-DF 64 file format.

stored and supplemented with the different embedded annotations.

\section{Waveform Data Format}

The waveform data (i.e., consumption data) must be defined in the Format chunk. This is inherited from the W64 format and consists of the following fields: i) sample size in bits $(8,16,24,32$ or 64 bits); and ii) number of individual channels (greater or equal to 1).

Additionally, all the sub-chunks defined in the Config list chunk are mandatory. More precisely: i) timezone (the time zone of the location where the data was collected), ii) timestamp (the Unix timestamp of the first sample in the waveform data), iii) sampling rate (the number of samples per second in the waveform data), and iv) calibration constants (zero or one for each waveform channel). The calibration constant chunks are associated to each channel in ascending order. For the model to be valid, the number of calibration chunks must be zero (i.e., no calibration is needed) or equal to the number of individual channels.

\section{Consumption Data}

The consumption data are represented using the Data, Missing Data List, and Missing Data chunks. The Missing Data List wraps sequences of Missing Data chunks to support datasets with missing data.

Waveform data are stored uncompressed in Data chunks. If only one metric needs to be represented (this is the case in most individual appliance and circuit ground-truth data), the samples are stored consecutively; otherwise the samples are stored interleaved. Each sample $S$ is represented by a value between -1 and 1 . Samples are stored in little-endian format (i.e., the least significant byte is stored first). The bits that represent the sample amplitude are stored in the most significant bits of $S$, and the remaining bits are set to zero. 


\begin{tabular}{|c|c|c|c|}
\hline Name & GUID & Description & Parent \\
\hline Config $^{a}$ & "CNFG" & List with file specific configuration chunks. & riff \\
\hline Timestamp $^{a}$ & "TMSP" & Unix timestamp of the first sample in the waveform data. & CNFG \\
\hline Timezone $^{a}$ & "TMZN" & Timezone of the place where the data was collected. & CNFG \\
\hline $\begin{array}{l}\text { Sampling } \\
\text { rate }^{a}\end{array}$ & "SPRT" & $\begin{array}{l}\text { Sampling rate of the waveform data (overwrites the original value in the format chunk if the } \\
\text { actual sampling rate is lower than } 1 \mathrm{~Hz} \text { ). }\end{array}$ & CNFG \\
\hline $\begin{array}{l}\text { Calibration } \\
\text { Constants }^{a}\end{array}$ & "CHCC" & $\begin{array}{l}\text { Calibration constants to recreate the original values of the waveform data. One constant for } \\
\text { each channel. }\end{array}$ & CNFG \\
\hline $\begin{array}{l}\text { Missing } \\
\text { Data List }\end{array}$ & "MDL " & List with missing data identifiers. & riff \\
\hline $\begin{array}{l}\text { Missing } \\
\text { Data }\end{array}$ & "MDAT" & Chunk containing a missing data identifier. & MDL \\
\hline Annotation & "ANNO" & Identifies a list that contains metadata and comment chunks. & riff \\
\hline Metadata & "META" , & This is metadata specific chunk. Must be contained in the Annotation chunk. & ANNO \\
\hline Comment & "COMT" & This is a comment specific chunk and must be specified within the Annotation chunk. & ANNO \\
\hline
\end{tabular}

Table 2. List of EMD-DF64 specific chunks

Handling Missing Data: Intervals with missing data are represented using the Missing Data chunk. Each of these chunks contains a JSON string with information about the timestamp when data is again available, and the number of the sample where this happens.

Listing 1. JSON Representation of a Missing Data Chunk.

\{

"Initial_Timestamp ": "2011-10-24 06:05:00.000",

"Initial_Sample": 139850 ,

\}

Timestamp Conversion: Since we do not store the timestamp of each individual sample, this has to be calculated in run-time. This is done using Equation (1), which returns a Unix timestamp in milliseconds:

$$
\text { unix_timestamp }=1000 \times \frac{\text { current_sample }- \text { initial_sample }}{f}+\text { initial_unix_timestamp }
$$

Where current_sample is the position of the sample of interest, initial_sample is the position of the first sample (if there are no missing data chunks the first sample is 1, otherwise it is the initial sample of the corresponding missing data chunk). initial_unix_timestamp is the unix timestamp of the first sample (if there are no missing data it is given by the Timezone chunk, otherwise it is calculated from the corresponding missing data chunk). Finally, $f$ is the sampling rate of the waveform data.

Conversely, it is possible to convert a unix timestamp to a sample position. This is done using Equation (2):

$$
\text { position }=\frac{\text { actual_timestamp }- \text { initial_timestamp }}{\frac{1}{f} \times 1000}
$$

Where actual_timestamp is the timestamp in milliseconds to be mapped to an audio position, initial_timestamp is the timestamp in milliseconds of the first sample in the dataset, and $f$ is the sampling rate of the waveform data.

\section{Ground-Truth: Individual Appliance Activity}

Individual appliance activities correspond to the changes in the power consumption that are triggered by different appliance turning ON, OFF, or changing their working mode (e.g., low to high).

Individual appliance activity (i.e., power events), are embedded in the file using the Cue, Associated Data List and Label chunks. This is done as follows: 1) For each power event, an entry is added in the Cue chunk, 2) for each Cue chunk entry, a Label chunk is created and added to the Associated Data List chunk.

Each label chunk consists of a sample position in the waveform data and a JSON formatted string with the details of the respective activity. The sample position is calculated from the power event timestamp using equation 2: For example, the JSON in Listing 2 corresponds to a refrigerator activity that was mapped to position 19394633: 
Listing 2. JSON Representation of a Refrigerator Activity.

\{

"ID" : 1101

"Type": 1 ,

"Position": 19394633

"Timestamp": "2011-10-24 05:45:57.040",

"App_ID" : 111 ,

"App_Label": "Refrigerator"

\}

\section{Ground-Truth: User Activity}

User activities refer to actions that are performed involving the use of electric appliances, e.g., doing the laundry (involves clothes washer, clothes dryer and iron) or preparing a meal (oven, stove, microwave, aid choppers, blenders, etc.).

User activities are supplemented in the consumption data using the Cue, Associated Data List and Labeled Text chunks. This is done as follows: 1) For each user activity, an entry is added in the Cue chunk, 2) for each Cue chunk entry, a Labeled text chunk is created and added to the Associated Data List chunk.

Each labeled text chunk consists of a sample position in the waveform data, a duration in samples, and a JSON formatted string with the details of the respective activity. Listing 3 shows a JSON representing the "working on the computer" activity that involves using the desktop computer (App_ID: 1101), one monitor (App_ID: 1109) and a printer ((App_ID: 1203)). The duration in samples is obtained by subtracting the start from the end position of the activity. The sample positions are calculated from the timestamps using equation 2.

Listing 3. JSON Representation of a User Activity

\{

"ID" : 10020

"Activity_ID": 111,

"Activity_Label": "Working on the computer",

"Start_Position": 19394633,

"End_Position": 19394633,

"Start_Timestamp": "2011-10-24 05:45:57.040",

"End_Timestamp": "2011-10-24 06:23:18.056",

"Appliance_Activity_IDs": [1101, 1109, 1203],

"Total_Power": 1000

\}

RIFF Meta

Since EMD-DF64 is a direct application of the RIFF standard, it fully supports all the default RIFF metadata sub-chunks that are defined in the Info chunk. These are used to supplement general annotations, and include among others, Creator, Commissioner, Copyright, and Keywords. The RIFF metadata chunks supported in EMD-DF64 are listed in Table 3.

\begin{tabular}{|l|l|l|}
\hline Name & GUID & Description \\
\hline File Creator & "IART" & The name of the file creator. \\
\hline Commissioner & "IMCS" & The name of the dataset commissioner. \\
\hline Comments & "ICMT" & Free text comment. \\
\hline Copyright & "ICOP" & Dataset copyright notice. \\
\hline Creation Data & "ICRD" & Data of dataset creation. \\
\hline Keywords & "IKEY" & A list of keywords to describe the dataset content. \\
\hline Name & "INAM" & The dataset name. \\
\hline Product & "IPRD" & Original purpose of the dataset. \\
\hline Subject & "ISBJ" & Contents of the file (e.g., current and voltage waveforms). \\
\hline Software & "ISFT" & Name of the software that was used to create the file. \\
\hline
\end{tabular}

Table 3. List of RIFF metadata chunks supported by EMD-DF64.

\section{Local Metadata}

Local metadata annotations can be used for instance to supplement datasets with details like the instant when a new appliance is added or removed from the electric circuit. Each local annotation is associated to a position in the waveform data and its content is encoded in a JSON string as shown in Figure 4.7. Local metadata are used to supplement specific consumption 
samples with custom annotations. For instance, when a new appliance is added or removed from the electric circuit as shown in JSON Listing 4.

A note chunk consists of a sample position and a JSON formatted string. Notes are created using the Cue, Additional Data List and Note chunks as follows: 1) For each local annotation, an entry is added to the Cue chunk, 2) for each entry in the Cue chunk, a Note chunk is added to the Associated Data List chunk.

Listing 4. JSON Representation of a Local Metadata

\{

"ID" : 1101 ,

"Position": 19394633,

"Timestamp": "2011-10-24 05:45:57.040",

"Text": "New refrigerator was added"

\}

\section{General Metadata}

These custom chunks can be used to enrich datasets with custom metadata. They are added using the Annotation List and Metadata chunks.

The content of such chunks do not follow any specific rule, yet it must be encoded in JSON and always include the ID and Label fields. EMD-DF64 fully supports three different custom metadata types: i) appliances; ii) user activities; and iii) NILM metadata project annotations.

Appliances metadata: keeps a list of the appliances that co-exist in the dataset, including the appliance characteristics like brand, model, energy consumption and energy efficiency rating. Listing 5 shows a possible JSON representation of the appliances metadata chunk.

Listing 5. JSON Representation of Appliances Custom Metadata.

\{

"ID" : 10021

"Label": "Dataset Appliances",

"Appliances":

[

\{

"ID": 111 ,

"Label": "Refrigerator",

"Brand": "Some brand",

"Model": "Some model",

"Energy_Consumption": 200,

"Energy_Efficiency_Rating": "E"

\} ,

]

\}

User activities metadata: keeps a list of the user activities that are present in the dataset, including a list of the appliances that can be associated with each activity. An example is provided in Listing 6.

Listing 6. JSON Representation of User Activities Custom Metadata.

\{

"ID" : 10022

"Label": "User Activities",

"User_Activities":

[

"ID" : 111,

"Label": "Working on the computer",

\},

"Appliance_IDs": [110, 211, 105]

\}

]

NILM Metadata: It is also possible to supplement datasets with annotations from the NILM metadata project. To this end we have defined the NILM Metadata Project annotation that can be used to embed the content of the different YAML files that compose the NILM Metadata project, in a metadata chunk. An example annotation is provided in Listing Listing 7. 
Listing 7. JSON Representation of a NILM Metadata Annotation.

\{

"ID" : 10023

"Label": "NILM Metadata Project",

"Dataset": "YAML content",

"Meter_Devices": "YAML content",

"Building":

[

"Building_1": "YAML content",

"Building_2": "YAML content"

\}

\section{Comments}

Custom comment chunks consist of free form text and are created using the Annotation List and Comment chunks. These can be used to add any kind of comments, for example, add a comment containing the historic of previous performance evaluations results on that particular file or dataset. Another example would be, adding a comment regarding some external event that could have affected the data.

\section{JSON Schemas}

Since most of the annotation data will be done using the JSON format we have decided to use JSON schemas ${ }^{6}$ to describe the JSON data elements presented in the previous sub-section. Listing 8 shows a snippet of the JSON-Schema for the Config chunk.

Listing 8. JSON schema for the config chunk.

\{

"\$schema": "http:// json-schema.org/draft/2020-12/schema\#",

"title ": "Config",

"description":"The mandatory config chunk",

"type": "object",

"proprieties" : \{

"initial_timestamp": \{

"type" : "string ",

"description":"This is the initial date and time for this file"

\} ,

"calibration_constants": \{

"type" : " array",

"items": \} ,

"type " : "number"

"minItems" : 2 ,

"maxItems " : 2

\} ,

"required": ["initial_timestamp", "calibration_constants"]

\}

\section{Discussion}

EMD-DF64 was developed to help mitigate the lack of homogeneity across existing electricity consumption datasets, which poses significant challenges for researchers intending to use datasets comparatively.

$\mathrm{In}^{15}$, the authors offered 17 suggestions for improving the collection, storage, and provision of electricity datasets. Next, we briefly discuss how EMD-DF64 promotes adopting some of those suggestions from a data storage and representation point of view.

Suggestion 5: gaps and irregularities EMD-DF64 supports missing data by default. Furthermore, by indicating the timestamps with missing data, it is straightforward to implement data cleaning strategies such as data interpolation. Local metadata can also be used to annotate regions where data is out of distribution (e.g., considerable changes to the voltage signal).

Suggestion 10: annotation of traces of event/activity information EMD-DF64 supports embedded annotations for both appliances (labels) and user activities (labeled text) by default. Furthermore, local metadata (notes) can be used to embed information on relevant changes, e.g., addition/removal/replacement of an appliance or changes to the number of household members.

${ }^{6}$ JSON Schemas, http: //json-schema.org/ 


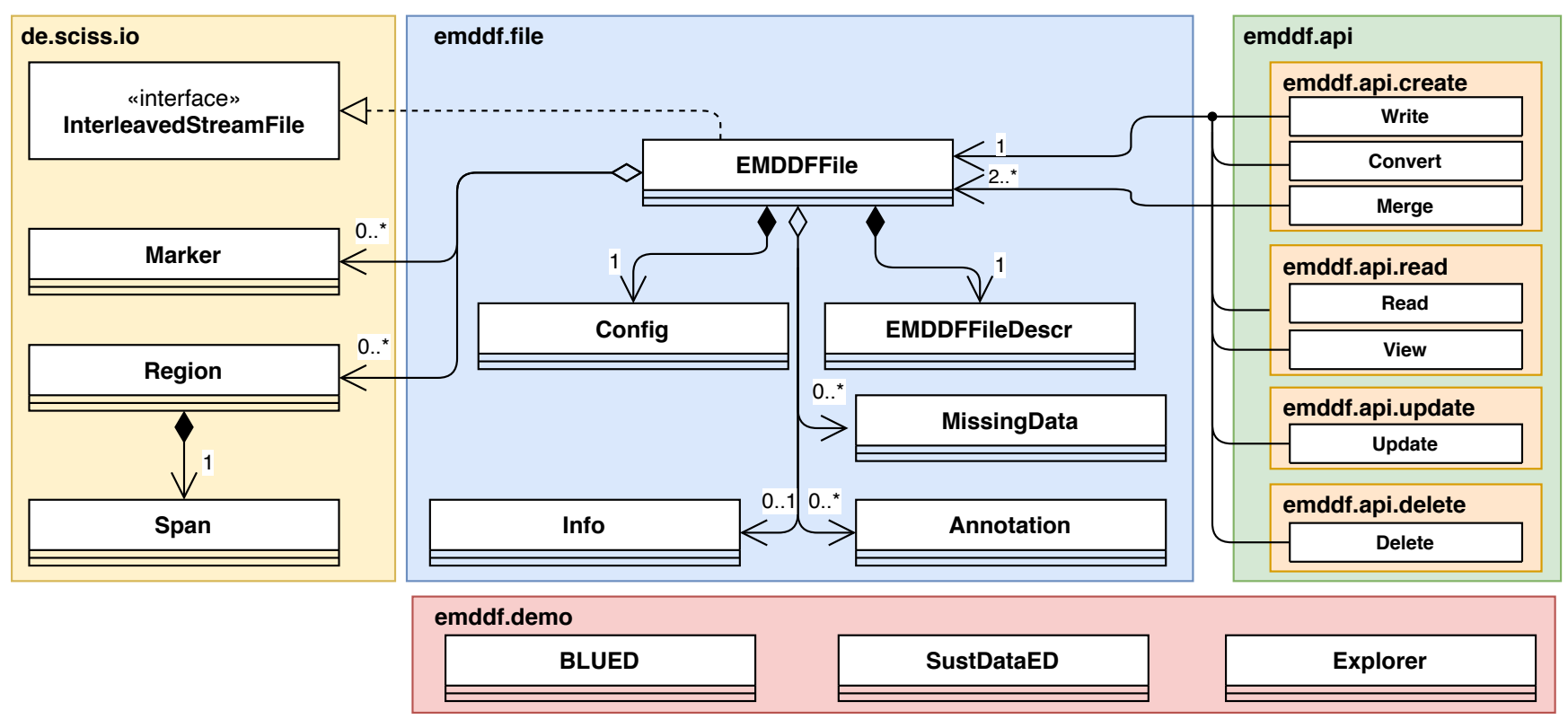

Figure 3. EMD-DF64 class diagram.

Suggestion 12: metadata formatted in reusable machine way/easy processing In EMD-DF64, metadata is represented using JSON, a widely used data-interchange format. Besides that, it is lightweight (e.g., compared to XML) and easy for humans to read and write. Furthermore, to remove ambiguity and errors when labeling the dataset, the annotations are validated using JSON schemas before being embedded in the data files.

Suggestion 14: compatibility of formats with software and compression By extending an audio format, it is possible to take advantage of the many audio libraries available in the different programming languages. For instance, in our particular case, we provide a Java implementation of EMD-DF64, which can be easily integrated with Python using the pyemddf ${ }^{7}$ package.

As for compression, EMD-DF64 is fully compatible with the WavPack audio compression format, which provides both lossless and hybrid (i.e., lossy with the possibility of complete restoration) compression modes. The compression ratio depends on the source material but generally is between $30 \%$ and $70 \%$. For example, using SustDataED, we have observed compression rates of 52\% for raw current and voltage waveforms sampled at $12.8 \mathrm{kHz}$. On the other hand, a rate of $70 \%$ was achieved for active power, reactive power, current, and voltage measurements sampled at $50 \mathrm{~Hz}$.

\section{Code Availability}

The present version of EMD-DF64 was implemented using the Java programming language. To this end, we extended the audio handling functionalities of ScissLib ${ }^{8}$. Figure 3 shows an overview of the EMD-DF64 class diagram. The EMD-DF64 implementation is released under the GNU General Public License (GPL) 3.0. Source-code and documentation are available in the project page at the Open Science Framework $(\mathrm{OSF})^{30}$.

\section{Data Availability}

All the data necessary to run the demo applications (BLUED and SustDataED) are available in the projects' OSF repository ${ }^{30}$. All the datasets used in the demo applications are available from the corresponding author on reasonable request. Please refer to the original publications for additional details.

\section{References}

1. Hart, G. Prototype nonintrusive appliance load monitor. Tech. Rep., MIT Energy Laboratory Technical Report, and Electric Power Research Institute Technical Report (1985).

\footnotetext{
${ }^{7}$ pyemddf, https://pypi.org/project/pyemddf/

${ }^{8}$ ScissLib, https://github.com/Sciss/ScissLib
} 
2. Zeifman, M. \& Roth, K. Nonintrusive appliance load monitoring: Review and outlook. IEEE Transactions on Consumer Electron. 57, 76-84, 10.1109/TCE.2011.5735484 (2011).

3. Pereira, L. \& Nunes, N. Performance evaluation in non-intrusive load monitoring: Datasets, metrics, and tools—a review. Wiley Interdiscip. Rev. Data Min. Knowl. Discov. 8, e1265, 10/gfb8gr.

4. Himeur, Y., Alsalemi, A., Bensaali, F. \& Amira, A. Building power consumption datasets: Survey, taxonomy and future directions. Energy Build. 227, 110404, 10.1016/j.enbuild.2020.110404.

5. Gao, J., Giri, S., Kara, E. C. \& Bergés, M. PLAID: A Public Dataset of High-resoultion Electrical Appliance Measurements for Load Identification Research: Demo Abstract. In Proceedings of the 1st ACM Conference on Embedded Systems for Energy-Efficient Buildings, BuildSys '14, 10.1145/2674061.2675032 (ACM, New York, NY, USA, 2014).

6. Makonin, S., Ellert, B., Bajić, I. V. \& Popowich, F. Electricity, water, and natural gas consumption of a residential house in Canada from 2012 to 2014. Sci. Data 3, 160037, 10.1038/sdata.2016.37 (2016).

7. Murray, D., Stankovic, L. \& Stankovic, V. An electrical load measurements dataset of United Kingdom households from a two-year longitudinal study. Sci. Data 4, 160122, 10/f9k7k9 (2017). 00029.

8. Kriechbaumer, T. \& Jacobsen, H.-A. BLOND, a building-level office environment dataset of typical electrical appliances. Sci. Data 5, 180048, 10.1038/sdata.2018.48 (2018).

9. Batra, N., Gulati, M., Singh, A. \& Srivastava, M. B. It's Different: Insights into Home Energy Consumption in India. In Proceedings of the 5th ACM Workshop on Embedded Systems For Energy-Efficient Buildings, BuildSys'13, 3:1-3:8, 10.1145/2528282.2528293 (ACM, New York, NY, USA, 2013).

10. Kelly, J. \& Knottenbelt, W. The UK-DALE dataset, domestic appliance-level electricity demand and whole-house demand from five UK homes. Sci. Data 2, 150007, 10.1038/sdata.2015.7 (2015).

11. Kahl, M., UI Haq, A., Kriechbaumer, T. \& Hans-Arno, J. WHITED - A Worldwide Household and Industry Transient Energy Data Set. In 3rd International NILM Workshop (Vancouver, BC, Canada, 2016).

12. Pereira, L., Nunes, N. \& Bergés, M. SURF and SURF-PI: A File Format and API for Non-intrusive Load Monitoring Public Datasets. In Proceedings of the 5th International Conference on Future Energy Systems, e-Energy '14, 225-226, 10.1145/2602044.2602078 (ACM, New York, NY, USA, 2014).

13. Ribeiro, M., Pereira, L., Quintal, F. \& Nunes, N. SustDataED: A Public Dataset for Electric Energy Disaggregation Research. In Proceedings of ICT for Sustainability 2016, Advances in Computer Science Research, 244-245, 10.2991/ ict4s-16.2016.36 (Atlantis Press, Amsterdam, The Netherlands, 2016).

14. Völker, B., Scholl, P. M. \& Becker, B. Semi-Automatic Generation and Labeling of Training Data for Non-intrusive Load Monitoring. In Proceedings of the Tenth ACM International Conference on Future Energy Systems, e-Energy '19, 17-23, 10/gf32w9 (ACM, 2019).

15. Klemenjak, C. et al. Electricity Consumption Data Sets: Pitfalls and Opportunities. In Proceedings of the 6th ACM International Conference on Systems for Energy-Efficient Buildings, Cities, and Transportation, BuildSys '19, 159-162, 10/ggfsnw (ACM, New York, NY, USA, 2019).

16. Kelly, J. \& Knottenbelt, W. Metadata for Energy Disaggregation. In 2014 IEEE 38th International Computer Software and Applications Conference Workshops, 578-583, 10.1109/COMPSACW.2014.97 (2014).

17. Batra, N. et al. NILMTK: An Open Source Toolkit for Non-intrusive Load Monitoring. In Proceedings of the 5th International Conference on Future Energy Systems, e-Energy '14, 265-276, 10.1145/2602044.2602051 (ACM, New York, NY, USA, 2014).

18. Paris, J., Donnal, J. S. \& Leeb, S. B. NilmDB: The Non-Intrusive Load Monitor Database. IEEE Transactions on Smart Grid 5, 2459-2467, 10.1109/TSG.2014.2321582.

19. Pereira, L. EMD-DF: A Data Model and File Format for Energy Disaggregation Datasets. In Proceedings of the 4th ACM International Conference on Systems for Energy-Efficient Built Environments (ACM, Delft, The Netherlands, 2017).

20. Pereira, L., Quintal, F., Nunes, N. \& Bergés, M. The Design of a Hardware-software Platform for Long-term Energy Eco-feedback Research. In ACM SIGCHI Symposium on Engineering Interactive Computing Systems, EICS '12, 221-230, 10.1145/2305484.2305521 (ACM, Copenhagen, Denmark, 2012).

21. Kriechbaumer, T., Jorde, D. \& Jacobsen, H.-A. Waveform Signal Entropy and Compression Study of Whole-Building Energy Datasets. In Proceedings of the Tenth ACM International Conference on Future Energy Systems, 58-67, 10/gf32xh (ACM, 2019). 00000. 
22. Anderson, K. et al. BLUED: A fully labeled public dataset for event-based non-intrusive load monitoring research. In Proceedings of the 2nd KDD Workshop on Data Mining Applications in Sustainability (SustKDD), 1-5 (2012).

23. Makonin, S., Wang, Z. J. \& Tumpach, C. RAE: The Rainforest Automation Energy Dataset for Smart Grid Meter Data Analysis. Data 3, 8, 10.3390/data3010008 (2018).

24. Medico, R. et al. A voltage and current measurement dataset for plug load appliance identification in households. Sci. Data 7, 1-10, 10.1038/s41597-020-0389-7 (2020).

25. Reinhardt, A. et al. On the accuracy of appliance identification based on distributed load metering data. In 2012 Sustainable Internet and ICT for Sustainability (SustainIT), 1-9 (2012).

26. Monacchi, A., Egarter, D., Elmenreich, W., D’Alessandro, S. \& Tonello, A. M. GREEND: An energy consumption dataset of households in Italy and Austria. In 2014 IEEE International Conference on Smart Grid Communications (SmartGridComm), 511-516, 10.1109/SmartGridComm.2014.7007698 (Venice, Italy, 2014).

27. Reinhardt, A. \& Klemenjak, C. Device-Free User Activity Detection using Non-Intrusive Load Monitoring: A Case Study. In Proceedings of the 2nd ACM Workshop on Device-Free Human Sensing, DFHS'20, 1-5, 10.1145/3427772.3429391 (Association for Computing Machinery).

28. Bousbiat, H., Klemenjak, C., Leitner, G. \& Elmenreich, W. Augmenting an Assisted Living Lab with Non-Intrusive Load Monitoring. In 2020 IEEE International Instrumentation and Measurement Technology Conference (I2MTC), 1-5, 10.1109/I2MTC43012.2020.9128406.

29. Multimedia Programming Interface and Data Specifications 1.0. Tech. Rep., IBM Corporation and Microsoft Corporation, USA (1991).

30. Pereira, L., Pereira, M. \& Velosa, N. EMD-DF64: A 64-Bit File Format for Energy Monitoring and Disaggregation Datasets, 10.17605/OSF.IO/D7EBX (2021).

\section{Author contributions statement}

Conceptualization, L.P.; methodology, L.P.; research, L.P., N.V. and M.P.; development, L.P., N.V. and M.P.; original draft preparation, L.P., N.V., and M.P.; review and editing, B.V.; visualization, L.P..

\section{Competing interests}

The authors declare no competing interests. 